\title{
A combinatorial algorithm to compute presentations of mapping-class groups of orientable surfaces with one boundary component
}

\author{
Lluís Bacardit*
}

\begin{abstract}
We give an algorithm which computes a presentation for a subgroup, denoted $\mathcal{A} \mathcal{M}_{g, 1, p}$, of the automorphism group of a free group. It is known that $\mathcal{A} \mathcal{M}_{g, 1, p}$ is isomorphic to the mapping-class group of an orientable genus- $g$ surface with one boundary component and $p$ punctures. We define a variation of Auter space.

2010 Mathematics Subject Classification. Primary: 57N05, 20F05; Secondary: 20F28, 20F34.

Key words. Mapping-class groups, presentations, automorphism groups, Auter space.
\end{abstract}

\section{Introduction}

Let $S$ be an orientable genus- $g$ surface with $b$ boundary components and $p$ punctures. We denote by $\mathcal{M}(S)$ the group of isotopy classes of orientation-preserving homeomorphisms of $S$ which permute the set of punctures and pointwise fix the boundary components. Since the group $\mathcal{M}(S)$ only depends, up to isomorphism, on the genus $g$ of $S$, the number $b$ of boundary components of $S$ and the number $p$ of punctures of $S$, we denote $\mathcal{M}(S)$ by $\mathcal{M}_{g, b, p}$. We call $\mathcal{M}_{g, b, p}$ the mapping-class group of $S$.

Presentations for $\mathcal{M}_{g, b, p}$ were obtained after a sequence of papers started by Hatcher and Thurston [11], and followed by Harer [10], Wajnryb [17], [18]; Matsumoto [14] and, Labruère and Paris [13]. For $p=0$, Gervais [9] used [17] to deduce another presentations for $\mathcal{M}_{g, b, 0}$. Before [11] very little was known about

*The research was funded by MIC (Spain) through Project MTM2008-01550. 
the presentation of $\mathcal{M}_{g, b, p}$. Birman and Hilden [4] gave a presentation for $\mathcal{M}_{2,0,0}$, and $\mathrm{McCool}$ [15] proved that $\mathcal{M}_{g, b, p}$ is finitely presented.

Benvenuti [3] uses a variation of the curve complex, called ordered curve complex, to obtain presentations for $\mathcal{M}_{g, b, p}$ from an inductive process. This inductive process starts from presentation for the sphere and the torus with "few" boundary components and/or punctures. Hirose [12] uses the curve complex and induction on $g$ and $b$ to deduce Gervais presentation. Both of these papers are independent of [11.

Our algorithm is independent of [11. We feel that our point of view goes back to McCool [15. Section 7 contains the presentation given by our algorithm. This presentation has generators $z e_{i}, z e_{i} e_{j}$ where $z$ ranges over a finite set $\mathcal{L}$ and $e_{i}, e_{j}$ range over $z$. There are three type of relations:

(a). $z e_{i}=1, z e_{i^{\prime}} e_{j^{\prime}}=1$, for some generators $z e_{i}, z e_{i^{\prime}} e_{j^{\prime}}$.

(b). $z_{1} e_{i} e_{j}=z_{2} e_{i^{\prime}} e_{j^{\prime}}$, for some generators $z_{1} e_{i} e_{j}, z_{2} e_{i^{\prime}} e_{j^{\prime}}$.

(c). $z e_{i} \cdot z e_{i} e_{j}=z e_{j} \cdot z e_{j} e_{i}$, for every generator $z e_{i} e_{j}$.

Armstrong, Forrest and Vogtmann [1 give a new presentation for $\operatorname{Aut}\left(F_{n}\right)$, the automorphism group of the free group of rank $n$. This presentation for $\operatorname{Aut}\left(F_{n}\right)$ is obtained by studying the action of $\operatorname{Aut}\left(F_{n}\right)$ on a subcomplex of the spine of Auter space. Following Armstrong, Forrest and Vogtmann [1, we obtain our algorithm by studying the action of an algebraic analogous of $\mathcal{M}_{g, 1, p}$ on a subcomplex of the spine of a variation of Auter space.

\section{Preliminaries}

Throughout the paper $n$ will be a non-negative integer, $F_{n}$ will be a free group of rank $n, \operatorname{Aut}\left(F_{n}\right)$ will be the automorphism group of $F_{n}$ and $\operatorname{Out}\left(F_{n}\right)$ will be the automorphism group of $F_{n}$ modulo inner automorphisms. Given $v, w \in F_{n}$, we denote by $[v, w]$ the element $v^{-1} w^{-1} v w$ of $F_{n}$. We denote by $[w]$ the conjugacy class of $w$.

Let $S$ be an orientable genus- $g$ surface with $b$ boundary components and $p$ punctures. A homeomorphism $f$ of $S$ which fixes the basepoint of $\pi_{1}(S)$ and permutes the set of punctures of $S$ induces an automorphism $f_{*} \in \operatorname{Aut}\left(\pi_{1}(S)\right)$. The isotopy class of $f$ defines an automorphism of $\pi_{1}(S)$ up to inner automorphisms, that is, an element of $\operatorname{Out}\left(\pi_{1}(S)\right)$. For $(b, p)=(0,0)$, by Dehn-Nielsen-Baer Theorem, $\mathcal{M}_{g, 0,0}$ is isomorphic to a index 2 subgroup of $\operatorname{Out}\left(\pi_{1}(S)\right)$. For $(g, p) \neq(0,0)$ by a modification of Dehn-Nielsen-Baer Theorem, $\mathcal{M}_{g, b, p}$ is isomorphic to an infinite index subgroup of $\operatorname{Out}\left(\pi_{1}(S)\right)$.

Suppose now $b=1$, that is, $S$ has exactly one boundary component. If we choose the basepoint of $\pi_{1}(S)$ to be a boundary point of $S$ and we restrict 
ourselves to homeomorphisms of $S$ which pointwise fix the boundary, then the isotopy class of a homeomorphism of $S$ defines an element of $\operatorname{Aut}\left(\pi_{1}(S)\right)$. Since $S$ has one boundary component, the fundamental group of $S$ is a free group. We denote by

$$
\Sigma_{g, 1, p}=\left\langle x_{1}, y_{1}, x_{2}, y_{2}, \ldots, x_{g}, y_{g}, t_{1}, t_{2}, \ldots, t_{p} \mid\right\rangle
$$

a presentation of $\pi_{1}(S, *)$ where $*$ is a boundary point of $S$, for every $1 \leq k \leq$ $p$ the generator $t_{k}$ represents a loop around the $k$-th puncture of $S$ and the word $\left[x_{1}, y_{1}\right]\left[x_{2}, y_{2}\right] \cdots\left[x_{g}, y_{g}\right] t_{1} t_{2} \cdots t_{p}$ represents a loop around the boundary component of $S$.

2.1 Definition. We denote by $\mathcal{A M}_{g, 1, p}$ the subgroup of $\operatorname{Aut}\left(\Sigma_{g, 1, p}\right)$ consisting of automorphisms of $\Sigma_{g, 1, p}$ which fix the word $\left[x_{1}, y_{1}\right]\left[x_{2}, y_{2}\right] \cdots\left[x_{g}, y_{g}\right] t_{1} t_{2} \cdots t_{p}$ of $\Sigma_{g, 1, p}$ and fix the set of conjugacy classes $\left[t_{1}^{-1}\right],\left[t_{2}^{-1}\right], \ldots,\left[t_{p}^{-1}\right]$ of $\Sigma_{g, 1, p}$.

Using a modification of Dehn-Nielsen-Baer Theorem, it can be proved that $\mathcal{M}_{g, 1, p}$ is isomorphic to $\mathcal{A M}_{g, 1, p}$, see [8] with some changes of notation and some different conventions. We call $\mathcal{A M}_{g, 1, p}$ the algebraic mapping-class group of an orientable genus- $g$ surface with one boundary component and $p$ punctures.

\section{Auter space $\mathbb{A}_{n}$}

3.1 Definition. Let $\left(\Gamma, v_{0}, \phi\right)$ be a 3 -tuple such that

1. $\Gamma$ is a finite connected graph with no separating edges.

2. $\Gamma$ is a metric graph with total volume 1 .

3. $v_{0}$ is a distinguished vertex of $\Gamma$.

4. Every vertex of $\Gamma$ but $v_{0}$ has valence at least $3 ; v_{0}$ has valence at least 2 .

5. $\phi: \pi_{1}\left(\Gamma, v_{0}\right) \rightarrow F_{n}$ is an isomorphism called "marking".

A point in $\mathbb{A}_{n}$ is an equivalence class of 3 -tuples $\left(\Gamma, v_{0}, \phi\right)$, where $\left(\Gamma, v_{0}, \phi\right)$ is equivalent to $\left(\Gamma^{\prime}, v_{0}^{\prime}, \phi^{\prime}\right)$ if there exists an isometry $h: \Gamma \rightarrow \Gamma$ such that $h\left(v_{0}\right)=v_{0}^{\prime}$ and the isomorphism $h_{*}: \pi_{1}\left(\Gamma, v_{0}\right) \rightarrow \pi_{1}\left(\Gamma^{\prime}, v_{0}^{\prime}\right)$ satisfies $\phi=\phi^{\prime} \circ h_{*}$.

We call $\mathbb{A}_{n}$ Auter space.

Auter space $\mathbb{A}_{n}$ was introduced by Hatcher and Vogtmann [2] as an analogous for $\operatorname{Aut}\left(F_{n}\right)$ of Outer space. Often in the literature the marking is defined as $\phi^{-1}: F_{n} \rightarrow \pi_{1}\left(\Gamma, v_{0}\right)$.

If $\Gamma$ has $k+1$ edges, then $\left(\Gamma, v_{0}, \phi\right)$ defines an open $k$-simplex of $\mathbb{A}_{n}$ denoted $\sigma\left(\Gamma, v_{0}, \phi\right)$. We can obtain $\sigma\left(\Gamma, v_{0}, \phi\right)$ by varying the length of the edges of $\Gamma$. The $k$-simplex $\sigma\left(\Gamma, v_{0}, \phi\right)$ can be parametrized by $\Delta^{k}$, the standard open $k$-simplex of $\mathbb{R}^{k}$, as follows: $\left(\Gamma_{s}, v_{0}, \phi\right) \in \sigma\left(\Gamma, v_{0}, \phi\right)$ is the point of $\mathbb{A}_{n}$ such that 
the length of the edges of $\Gamma_{s}$ equal the barycentric coordinates of $s \in \Delta^{k}$. It is important that $\Delta^{k}$ is open. Since a non-trivial isometry of $\Gamma$ permutes same edges of $\Gamma$, such an isometry gives a non-trivial element of $H_{1}(\Gamma)$. Hence, every $s \in \Delta$ defines a different point of $\sigma\left(\Gamma, v_{0}, \phi\right)$.

Some faces of $\sigma\left(\Gamma, v_{0}, \phi\right)$ belong to $\mathbb{A}_{n}$. If an edge of $\Gamma$ is incident to two different vertices, then we can reduce the length of that edge to zero, and increase the length of the other edges, to obtain a new graph $\Gamma^{\prime}$ with one edge minus. We say that we have collapsed one edge of $\Gamma$. We have a quotient map $\Gamma \rightarrow \Gamma^{\prime}$ which defines a point $\left(\Gamma^{\prime}, v_{0}^{\prime}, \phi^{\prime}\right)$ of $\mathbb{A}_{n}$. We say that $\sigma\left(\Gamma^{\prime}, v_{0}^{\prime}, \phi^{\prime}\right)$ is a face of $\sigma\left(\Gamma, v_{0}, \phi\right)$. Faces of $\sigma\left(\Gamma^{\prime}, v_{0}^{\prime}, \phi^{\prime}\right)$ are faces of $\sigma\left(\Gamma, v_{0}, \phi\right)$. We cannot collapse an edge which is incident to a unique vertex. Hence, some face of $\sigma\left(\Gamma, v_{0}, \phi\right)$ are missing. In particular, $\mathbb{A}_{n}$ is not a simplicial complex.

There exists a deformation retract, denoted $\mathrm{SA}_{n}$, of $\mathbb{A}_{n}$ which is a simplicial complex. We can define $\mathrm{SA}_{n}$ as follows. For every simplex of $\mathbb{A}_{n}$, there exists a vertex of $\mathrm{SA}_{n}$. Two vertices of $\mathrm{SA}_{n}$ expand an edge if the simplex of $\mathbb{A}_{n}$ which defines one of the two vertices of $\mathrm{SA}_{n}$ is a face of the simplex of $\mathbb{A}_{n}$ which defines the other vertex of $\mathrm{SA}_{n} ; i+1$ vertices of $\mathrm{SA}_{n}$ expand a $i$-simplex of $\mathrm{SA}_{n}$ if every pair of vertices expand an edge.

There exists a natural inclusion of $\mathrm{SA}_{n}$ into $\mathbb{A}_{n}$ by sending every vertex of $\mathrm{SA}_{n}$ to the barycenter of the corresponding simplex, and every $i$-simplex of $\mathrm{SA}_{n}$ to the convex hull of the corresponding barycenters. This inclusion is a deformation retract. See [2].

Collapsing an edge of $\Gamma$ has an inverse process which splits a vertex of $\Gamma$ into two new vertices, and, the two new vertices are joined by a new edge. Often in the literature splitting of a vertex is called blowing up an edge. If $\tilde{\Gamma}$ is obtained from $\Gamma$ by splitting a vertex, then we can identify, in a natural way, every edge of $\Gamma$ with an edge of $\tilde{\Gamma}$. Collapsing the only edge of $\tilde{\Gamma}$ which is not identified with an edge of $\Gamma$ we obtain $\Gamma$. There exists a quotient map $\tilde{\Gamma} \rightarrow \Gamma$. If $\tilde{\Gamma}$ is obtained from $\Gamma$ by splitting a vertex different from $v_{0}$, then the quotient map $\tilde{\Gamma} \rightarrow \Gamma$ defines a point $\left(\tilde{\Gamma}, \tilde{v}_{0}, \tilde{\phi}\right)$ of $\mathbb{A}_{n}$ If $\tilde{\Gamma}$ is obtained from $\Gamma$ by splitting $v_{0}$, then the point $\left(\tilde{\Gamma}, \tilde{v}_{0}, \tilde{\phi}\right)$ of $\mathbb{A}_{n}$ depends of the election, between the two possibilities, of the new distinguished vertex $\tilde{v}_{0}$. The simplex $\sigma\left(\Gamma, v_{0}, \phi\right)$ is a face of $\sigma\left(\tilde{\Gamma}, \tilde{v}_{0}, \tilde{\phi}\right)$.

We give a combinatorial definition of the topological type of $\Gamma$, that is, $\Gamma$ when we forget its metric. When we forget the metric of $\Gamma$ we can see $\left(\Gamma, v_{0}, \phi\right)$ as a vertex of $\mathrm{SA}_{n}$, in fact, the vertex of $\mathrm{SA}_{n}$ defined by the simplex $\sigma\left(\Gamma, v_{0}, \phi\right)$ of $\mathbb{A}_{n}$. We translate to our combinatorial definition the processes of collapsing an edges and splitting a vertex. Our combinatorial definition of the topological type of $\Gamma$ is different from the one given in [6].

\subsection{Definition. Let}

1. $V(\Gamma)$ be the set of vertices of $\Gamma$.

2. $E(\Gamma)$ be the set of edges of $\Gamma$. 
3. $\bar{E}(\Gamma)=\{\bar{e} \mid e \in E(\Gamma)\}$ be a set disjoint with $E(\Gamma)$.

We extend - to an involution of $E(\Gamma) \cup \bar{E}(\Gamma)$. We fix an orientation of every edge of $\Gamma$. We say that $e \in E(\Gamma)$ starts at $v_{1} \in V(\Gamma)$ and finishes at $v_{2} \in V(\Gamma)$ if $e$ is incident to $v_{1}$ and $v_{2}$; and $e$ is oriented from $v_{1}$ to $v_{2}$. In this case we say that $\bar{e}$ starts at $v_{2}$ and finishes at $v_{1}$.

Given $v \in V(\Gamma)$, we define the following subset of $E(\Gamma) \cup \bar{E}(\Gamma)$.

$$
v^{*}=\{a \in E(\Gamma) \cup \bar{E}(\Gamma) \mid a \text { starts at } v\} .
$$

We set $V^{*}(\Gamma)=\left\{v^{*} \mid v \in V(\Gamma)\right\}$.

The topological type of $\Gamma$ is completely determined by $\left(V(\Gamma), E(\Gamma), V^{*}(\Gamma)\right)$.

Notice that $v^{*}$ is the star of $v \in V(\Gamma)$ and $V^{*}(\Gamma)$ is a partition of $E(\Gamma) \cup \bar{E}(\Gamma)$. Condition 1 of Definition 3.1 can be translated by saying that $E(\Gamma)$ is finite and, for every $v \in V(\Gamma)$, there exist $a, b \in v^{*}$ such that $a \neq b, \bar{b}$ and $\bar{a}, \bar{b} \notin v^{*}$. Condition 4 of Definition 3.1 can be translated by saying that for every $v \in$ $V(\Gamma)-\left\{v_{0}\right\}, v^{*}$ has at least 3 elements; $v_{0}^{*}$ has at least 2 elements.

3.3 Definition. Let $e \in E(\Gamma)$ such that $e \in v_{1}^{*}, \bar{e} \in v_{2}^{*}$, where $v_{1}, v_{2} \in V(\Gamma)$ and $v_{1} \neq v_{2}$. We can collapse $e$. When we collapse the edge $e$ we have a graph with topological type

$$
\left(V(\Gamma) \cup\{v\}-\left\{v_{1}, v_{2}\right\}, E(\Gamma)-\{e\}, V^{*}(\Gamma) \cup\left\{v^{*}\right\}-\left\{v_{1}^{*}, v_{2}^{*}\right\}\right)
$$

where $v \notin V(\Gamma)$ and $v^{*}=v_{1}^{*} \cup v_{2}^{*}-\{e, \bar{e}\}$.

3.4 Definition. Let $v \in V(\Gamma)-\left\{v_{0}\right\}$ and $A, B$ a partition of $v^{*}$ such that both $A$ and $B$ have at least two elements, there exists $a \in A$ such that $\bar{a} \notin A$ and there exists $b \in B$ such that $\bar{b} \notin B$. When we split the vertex $v$ with respect to $A$ and $B$ we have a graph with topological type

$$
\left(V(\Gamma) \cup\left\{v_{1}, v_{2}\right\}-\{v\}, E(\Gamma) \cup\{e\}, V^{*}(\Gamma) \cup\left\{v_{1}^{*}, v_{2}^{*}\right\}-\left\{v^{*}\right\}\right),
$$

where $v_{1}, v_{2} \notin V(\Gamma), e \notin E(\Gamma), v_{1}^{*}=A \cup\{e\}$ and $v_{2}^{*}=B \cup\{\bar{e}\}$. To split $v_{0}$ we have to choose between $v_{1}$ or $v_{2}$ as the new distinguished vertex. Since the distinguished vertex can have valence two, the subset of $v_{0}^{*}$ corresponding to the new distinguished vertex may have only one element.

\section{Ordered Auter space ord $\mathbb{A}_{g, p}$}

Our motivation for defining ordered Auter space is that when a graph is embedded into an orientable surface, the star of every vertex of the graph which is mapped to an interior point of the surface gets a cyclic order, and, the star of a vertex which is mapped to a boundary point of the surface gets a linear order. When we want to collapse an edge or to split a vertex we have to do it respecting the orders of the stars. 
4.1 Definition. Let $\left(\Gamma, v_{0}, \phi\right.$, ord $)$ be a 4 -tuple where $\left(\Gamma, v_{0}, \phi\right)$ satisfies conditions in Definition [3.1, ord is a linear order of $v_{0}^{*}$ and a cyclic order of $v^{*}$ for every $v \in V(\Gamma)-\left\{v_{0}\right\}$.

Suppose $V(\Gamma)=\left\{v_{0}, v_{1}, v_{2}, \ldots, v_{q}\right\}$ and

$$
\begin{aligned}
\operatorname{ord}\left(v_{0}^{*}\right) & =\left(a_{1}^{0}, a_{2}^{0}, \ldots, a_{r_{0}}^{0}\right), \\
\operatorname{ord}\left(v_{1}^{*}\right) & =\left(a_{1}^{1}, a_{2}^{1}, \ldots, a_{r_{1}}^{1}\right), \\
\operatorname{ord}\left(v_{2}^{*}\right) & =\left(a_{1}^{2}, a_{2}^{2}, \ldots, a_{r_{2}}^{2}\right), \\
& \vdots \\
\operatorname{ord}\left(v_{q}^{*}\right) & =\left(a_{1}^{q}, a_{2}^{q}, \ldots, a_{r_{q}}^{q}\right) .
\end{aligned}
$$

For $i \neq 0, \operatorname{since~} \operatorname{ord}\left(v_{i}^{*}\right)$ is cyclically ordered, the subindices of $\operatorname{ord}\left(v_{i}^{*}\right)$ are modulo $r_{i}$

We consider the following element of $\pi_{1}\left(\Gamma, v_{0}\right)$ and the following conjugacy classes of $\pi_{1}\left(\Gamma, v_{0}\right)$.

$$
\begin{aligned}
w_{0} & =b_{1}^{0} b_{2}^{0} \cdots b_{l_{0}}^{0} \\
{\left[w_{1}\right] } & =\left[b_{1}^{1} b_{2}^{1} \cdots b_{l_{1}}^{1}\right] \\
{\left[w_{2}\right] } & =\left[b_{1}^{2} b_{2}^{2} \cdots b_{l_{2}}^{2}\right], \\
& \vdots \\
{\left[w_{p}\right] } & =\left[b_{1}^{p} b_{2}^{p} \cdots b_{l_{p}}^{p}\right],
\end{aligned}
$$

where $b_{1}^{0}=a_{1}^{0}$, for every $1 \leq i \leq p, 1 \leq j \leq l_{i}$ the subsequence $\left(\bar{b}_{j}^{i}, b_{j+1}^{i}\right)$ appears in (4.1.1), $b_{l_{0}}^{0}=\bar{a}_{r_{q}}^{q}$, and every element of $E(\Gamma) \cup E(\Gamma)$ appears exactly once in (4.1.2).

We denote by $w\left(\Gamma, v_{0}\right.$, ord $)$ the set $\left\{w_{0},\left[w_{1}\right],\left[w_{2}\right], \ldots,\left[w_{p}\right]\right\}$.

4.2 Example. Let $\left(\Gamma, v_{0}\right.$, ord) be a 3-tuple where $V(\Gamma)=\left\{v_{0}, v_{1}, v_{2}\right\}, E(\Gamma)=$ $\left\{e_{1}, e_{2}, e_{3}, e_{4}, e_{5}\right\}$ and

$$
\begin{aligned}
& \operatorname{ord}\left(v_{0}^{*}\right)=\left(e_{1}, e_{2}\right), \\
& \operatorname{ord}\left(v_{1}^{*}\right)=\left(\bar{e}_{1}, e_{3}, \bar{e}_{3}, e_{4}, e_{5}\right), \\
& \operatorname{ord}\left(v_{2}^{*}\right)=\left(\bar{e}_{2}, \bar{e}_{5}, \bar{e}_{4}\right) .
\end{aligned}
$$

Then $w\left(\Gamma, v_{0}\right.$, ord $)=\left\{w_{0},\left[w_{1}\right],\left[w_{2}\right],\left[w_{3}\right]\right\}$ where

$$
\begin{aligned}
w_{0} & =e_{1} e_{3} e_{4} \bar{e}_{2}, \\
{\left[w_{1}\right] } & =\left[\bar{e}_{1} e_{2} \bar{e}_{5}\right], \\
{\left[w_{2}\right] } & =\left[\bar{e}_{3}\right], \\
{\left[w_{3}\right] } & =\left[\bar{e}_{4} e_{5}\right] .
\end{aligned}
$$

Notice that for every $v \in V(\Gamma)$, ord $\left(v^{*}\right)$ is completely determined by $w\left(\Gamma, v_{0}\right.$, ord $)$. 
4.3 Definition. Let $\left(\Gamma, v_{0}, \phi\right.$, ord $)$ be a 4 -tuple such that $w\left(\Gamma, v_{0}\right.$, ord $)$ has $p$ conjugacy classes.

We denote $\frac{n-p}{2}$ by $g$. We will see that $n-p$ is even. Hence, $g$ is a non-negative integer.

We define ord $\mathbb{A}_{g, p}$ as the space of equivalence classes of 4 -tuples $\left(\Gamma, v_{0}, \phi\right.$, ord $)$ such that $\phi: \pi_{1}\left(\Gamma, v_{0}\right) \rightarrow \Sigma_{g, 1, p}, w\left(\Gamma, v_{0}\right.$, ord $)=\left\{w_{0},\left[w_{1}\right],\left[w_{2}\right], \ldots,\left[w_{p}\right]\right\}$ and

$$
\begin{aligned}
\phi\left(w_{0}\right) & =\left[x_{1}, y_{1}\right]\left[x_{2}, y_{2}\right] \cdots\left[x_{g}, y_{g}\right] t_{1} t_{2} \cdots t_{p}, \\
\left\{\phi\left(\left[w_{1}\right]\right), \phi\left(\left[w_{2}\right]\right), \ldots, \phi\left(\left[w_{p}\right]\right)\right\} & =\left\{\left[t_{1}^{-1}\right],\left[t_{2}^{-1}\right], \ldots,\left[t_{p}^{-1}\right]\right\} .
\end{aligned}
$$

The 4-tuples $\left(\Gamma, v_{0}, \phi\right.$, ord $)$ and $\left(\Gamma^{\prime}, v_{0}^{\prime}, \phi^{\prime}\right.$, ord $\left.^{\prime}\right)$ represent the same point of $\operatorname{ord} \mathbb{A}_{g, p}$ if there exists an isometry $h: \Gamma \rightarrow \Gamma^{\prime}$ such that $h\left(v_{0}\right)=v_{0}^{\prime}$, the isomorphism $h_{*}: \pi_{1}\left(\Gamma, v_{0}\right) \rightarrow \pi_{1}\left(\Gamma^{\prime}, v_{0}^{\prime}\right)$ satisfies $\phi=\phi^{\prime} \circ h_{*}$, and $h: \Gamma \rightarrow \Gamma^{\prime}$ preserves the orders, that is, ord $\left(v^{*}\right)=\left(a_{1}, a_{2}, \ldots, a_{r}\right)$ implies $\operatorname{ord}^{\prime}\left(h(v)^{*}\right)=$ $\left(h\left(a_{1}\right), h\left(a_{2}\right), \ldots, h\left(a_{r}\right)\right)$ for every $v \in V(\Gamma)$.

We call ord $\mathbb{A}_{g, p}$ ordered Auter space.

We define ordSA $\mathbb{A}_{g, p}$ for ord $\mathbb{A}_{g, p}$ as we defined $\mathrm{SA}_{n}$ for $\mathbb{A}_{n}$. In particular,

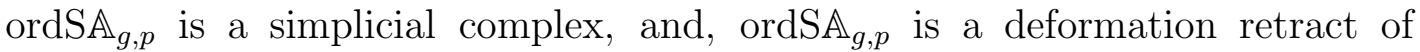
$\operatorname{ord} \mathbb{A}_{g, p}$.

The following definitions are based on Definition 3.3 and Definition [3.4, respectively.

4.4 Definition. Let $e \in E(\Gamma)$. Suppose $e=a_{k_{1}}^{i}, \bar{e}=a_{k_{2}}^{j}$, where $i \neq j$ and $1 \leq k_{1} \leq r_{i}, 1 \leq k_{2} \leq r_{j}$. Since $i \neq j$, we can collapse $e$. We can suppose $j \neq 0$. To adapt Definition 3.3 to ordSA $\mathbb{A}_{g, p}$ we set

$$
\begin{aligned}
\operatorname{ord}\left(v^{*}\right)=( & a_{k-1}^{i}, a_{k_{2}}^{i}, \ldots, a_{k_{1}-1}^{i}, \\
& a_{k_{2}+1}^{j}, a_{k_{2}+2}^{j}, \ldots, a_{r_{j}}^{j}, a_{1}^{j}, a_{2}^{j}, \ldots, a_{k_{2}-1}^{j}, \\
& \left.a_{k_{1}+1}^{i}, a_{k_{1}+2}^{i}, \ldots, a_{r_{1}}^{i}\right) .
\end{aligned}
$$

4.5 Example. Let $\left(\Gamma, v_{0}\right.$, ord $)$ be as in Example 4.2. When we collapse $e_{1}$ we obtain $\left(\Gamma^{\prime}, v_{0}^{\prime}, \operatorname{ord}^{\prime}\right)$ such that $v_{0}^{\prime}=v_{0}, V\left(\Gamma^{\prime}\right)=\left\{v_{0}, v_{2}\right\}, E\left(\Gamma^{\prime}\right)=\left\{e_{2}, e_{3}, e_{4}, e_{5}\right\}$ and

$$
\begin{aligned}
& \operatorname{ord}^{\prime}\left(v_{0}^{*}\right)=\left(e_{3}, \bar{e}_{3}, e_{4}, e_{5}, e_{2}\right), \\
& \operatorname{ord}^{\prime}\left(v_{2}^{*}\right)=\left(\bar{e}_{2}, \bar{e}_{5}, \bar{e}_{4}\right) .
\end{aligned}
$$

We have $w\left(\Gamma^{\prime}, v_{0}^{\prime}, \operatorname{ord}^{\prime}\right)=\left\{w_{0}^{\prime},\left[w_{1}^{\prime}\right],\left[w_{2}^{\prime}\right],\left[w_{3}^{\prime}\right]\right\}$ where

$$
\begin{aligned}
& w_{0}^{\prime}=e_{3} e_{4} \bar{e}_{2}, \\
& {\left[w_{1}^{\prime}\right]=\left[e_{2} \bar{e}_{5}\right],} \\
& {\left[w_{2}^{\prime}\right]=\left[\bar{e}_{3}\right],} \\
& {\left[w_{3}^{\prime}\right]=\left[\bar{e}_{4} e_{5}\right] .}
\end{aligned}
$$


Let $\left(\Gamma, v_{0}, \phi\right.$, ord $)$ be a vertex of ordSA $\mathbb{A}_{g, p}$. When we collapse an edge of $\Gamma$ according to Definition 4.4 we obtain $\left(\Gamma^{\prime}, v_{0}^{\prime}, \phi^{\prime}, \operatorname{ord}^{\prime}\right)$. As it is see in Example 4.5, $w\left(\Gamma^{\prime}, v_{0}^{\prime}, \phi^{\prime}, \operatorname{ord}^{\prime}\right)$ has $p$ conjugacy classes. Hence, $\left(\Gamma^{\prime}, v_{0}^{\prime}, \phi^{\prime}, \operatorname{ord}^{\prime}\right)$ is a vertex of $\operatorname{ordS} \mathbb{A}_{g, p}$.

4.6 Definition. Let $v \in V(\Gamma)$. Let $A, B$ be a partition of $v^{*}$. Suppose ord $\left(v^{*}\right)=$ $\left(a_{1}, a_{2}, \ldots, a_{r}\right)$ and $A=\left(a_{k_{1}}, a_{k_{1}+1}, \ldots, a_{k_{2}}\right)$, where $1 \leq k_{1}<k_{2} \leq r$. We can split the vertex $v$ with respect to $A, B$. To adapt Definition 3.4 to ordSA $\mathbb{A}_{g, p}$ we set

$$
\begin{aligned}
\operatorname{ord}\left(v_{1}^{*}\right)= & \left(e, a_{k_{1}}, a_{k_{1}+1}, \ldots, a_{k_{2}}\right) \\
\operatorname{ord}\left(v_{2}^{*}\right)= & \left(a_{1}, a_{2}, \ldots, a_{k_{1}-1},\right. \\
& \left.\bar{e}, a_{k_{2}+1}, a_{k_{2}+2}, \ldots a_{r}\right) .
\end{aligned}
$$

4.7 Example. Let $\left(\Gamma, v_{0}\right.$, ord $)$ be as in Example 4.2. When we split $v_{1}$ with respect to $\left\{\bar{e}_{3}, e_{4}\right\},\left\{\bar{e}_{1}, e_{3}, e_{5}\right\}$ we obtain $\left(\tilde{\Gamma}, \tilde{v}_{0}\right.$, ord $)$ such that $\tilde{v}_{0}=v_{0}, V(\tilde{\Gamma})=$ $\left\{v_{0}, v_{1,1}, v_{1,2}, v_{2}\right\}, E(\tilde{\Gamma})=\left\{e, e_{1}, e_{2}, e_{3}, e_{4}, e_{5}\right\}$ and

$$
\begin{aligned}
& \tilde{\operatorname{ord}}\left(v_{0}^{*}\right)=\left(e_{1}, e_{2}\right), \\
& \tilde{\operatorname{ord}}\left(v_{1,1}^{*}\right)=\left(e, \bar{e}_{3}, e_{4}\right) \text {, } \\
& \tilde{\operatorname{ord}}\left(v_{1,2}^{*}\right)=\left(\bar{e}_{1}, e_{3}, \bar{e}, e_{5}\right) \text {, } \\
& \operatorname{ord}\left(v_{2}^{*}\right)=\left(\bar{e}_{2}, \bar{e}_{5}, \bar{e}_{4}\right) \text {. }
\end{aligned}
$$

We have $w\left(\tilde{\Gamma}, \tilde{v}_{0}\right.$, ord $)=\left\{\tilde{w}_{0},\left[\tilde{w}_{1}\right],\left[\tilde{w}_{2}\right],\left[\tilde{w}_{3}\right]\right\}$ where

$$
\begin{aligned}
& \tilde{w}_{0}=e_{1} e_{3} e_{4} \bar{e}_{2}, \\
& {\left[\tilde{w}_{1}\right]=\left[\bar{e}_{1} e_{2} \bar{e}_{5}\right],} \\
& {\left[\tilde{w}_{2}\right]=\left[\bar{e}_{3} \bar{e}\right]} \\
& {\left[\tilde{w}_{3}\right]=\left[\bar{e}_{4} e e_{5}\right] .}
\end{aligned}
$$

Let $\left(\Gamma, v_{0}, \phi\right.$, ord $)$ be a vertex of ordSA $\mathbb{A}_{g, p}$. When we split a vertex of $\Gamma$ according to Definition 4.6 we obtain $\left(\tilde{\Gamma}, \tilde{v}_{0}, \hat{\phi}\right.$, ord $)$. As it is see in Example 4.7, $w\left(\tilde{\Gamma}, \tilde{v}_{0}, \tilde{\phi}\right.$, ord $)$ has $p$ conjugacy classes. Hence, $\left(\tilde{\Gamma}, \tilde{v}_{0}, \tilde{\phi}\right.$, ord $)$ is a vertex of $\operatorname{ordS} \mathbb{A}_{g, p}$.

Since a graph satisfying Definition 3.1 can have at most $3 n-2$ edges, the dimension of $\mathbb{A}_{n}$ is $3 n-3$. On the other hand, $\mathbb{A}_{n}$ is not a manifold. The dimension of ord $\mathbb{A}_{g, p}$ is $6 g+3 p-3$; ord $\mathbb{A}_{n}$ is a manifold.

By [2, PROPOSITION 2.1] $\mathbb{A}_{n}$ is contractible. Since $\mathrm{SA}_{n}$ is a deformation retract of $\mathbb{A}_{n}$, we see that $S \mathbb{A}_{n}$ is contractible. 
4.8 Proposition. ord $\mathbb{A}_{g, p}$ is contractible.

Hatcher and Vogtmann proof [2, PROPOSITION 2.1] using spheres complexes. It is not clear how to translate to the context of spheres complexes an ordered graph. On the other hand, the proof of Culler and Vogtmann [6] that Outer space is contractible can be applied to ord $\mathbb{A}_{g, p}$ : adding a basepoint is straightforward, all the geometric arguments in [6] can be applied to ord $\mathbb{A}_{g, p}$ respecting the orders as Definition 4.4 and Definition 4.6 and $\mathrm{McCool}$ [16], [7] proved that $\mathcal{A M}_{g, 1, p}$ is generated by Nielsen automorphism which "respect" the orders (recall that Nielsen automorphisms are a special case of Whitehead automorphisms).

Recall $n=2 g+p$. There exists a natural map ord $\mathbb{A}_{g, p} \rightarrow \mathbb{A}_{n}$ which "forgets" the ordering, that is, $\left(\Gamma, v_{0}, \phi\right.$, ord $) \mapsto\left(\Gamma, v_{0}, \phi\right)$. Recall that ord is completely determined by $w\left(\Gamma, v_{0}\right.$, ord $)$. Since $\phi: \pi_{1}\left(\Gamma, v_{0}\right) \rightarrow \Sigma_{g, 1, p}$ is an isomorphism, we have

$$
\begin{array}{r}
w\left(\Gamma, v_{0}, \text { ord }\right)=\left\{\phi^{-1}\left(\left[x_{1}, y_{1}\right]\left[x_{2}, y_{2}\right] \cdots\left[x_{g}, y_{g}\right] t_{1} t_{2} \cdots t_{p}\right),\right. \\
\left.\left.\left[\phi^{-1}\left(t_{1}^{-1}\right)\right],\left[\phi^{-1}\left(t_{2}^{-1}\right)\right], \ldots,\left[\phi^{-1}\left(t_{p}^{-1}\right)\right]\right)\right\}
\end{array}
$$

Hence, the natural map ord $\mathbb{A}_{g, p} \rightarrow \mathbb{A}_{n}$ is injective.

We want to see that $n-p$ is even.

For $n=1$, we have $p=1$ and $n-p=0$ is even. We do induction on $n$.

By Definition 4.4 we can collapse a maximal subtree of $\left(\Gamma, v_{0}\right.$, ord). Hence, we can suppose that $V(\Gamma)=\left\{v_{0}\right\}$. Put $\operatorname{ord}\left(v_{0}^{*}\right)=\left(a_{1}, a_{2}, \ldots a_{2 n}\right)$ and $w\left(\Gamma, v_{0}\right.$, ord $)=\left\{w_{0},\left[w_{1}\right],\left[w_{2}\right], \ldots,\left[w_{p}\right]\right\}$. Let $1 \leq j \leq 2 n$ such that $a_{j}=\bar{a}_{1}$. Then $w_{0}=a_{1} u$ in reduced form, for some $u \in F_{n}$. Let $\left(\Gamma^{\prime}, v_{0}^{\prime}, \operatorname{ord}^{\prime}\right)$ be obtained from $\left(\Gamma, v_{0}\right.$, ord) be deleting the edges $a_{1}, a_{j}$. We have $\operatorname{ord}^{\prime}\left(v_{0}^{\prime}\right)=$ $\left(a_{2}, a_{3}, \ldots, a_{j-1}, a_{j+1}, a_{j+2}, \ldots, a_{2 n}\right)$. We put $n^{\prime}=n-1$ the rank of $\pi_{1}\left(\Gamma^{\prime}, v_{0}^{\prime}\right)$ and $p^{\prime}$ the number of conjugacy classes of $w\left(\Gamma^{\prime}, v_{0}^{\prime}, \operatorname{ord}^{\prime}\right)$. By induction hypothesis $n^{\prime}-p^{\prime}$ is even.

If $w_{0}=a_{1} u^{\prime} a_{j} u^{\prime \prime}$ cyclically reduced, then $w\left(\Gamma^{\prime}, v_{0}^{\prime}, \operatorname{ord}^{\prime}\right)=\left\{u^{\prime \prime},\left[u^{\prime}\right],\left[w_{1}\right]\right.$, $\left.\left[w_{2}\right], \ldots,\left[w_{p}\right]\right\}$. Notice that $u^{\prime} \neq 1, u^{\prime \prime} \neq 1$ because $a_{1} u^{\prime} a_{j} u^{\prime \prime}$ is cyclically reduced. Hence, $p^{\prime}=p+1$ and $n-p=\left(n^{\prime}+1\right)-\left(p^{\prime}-1\right)=n^{\prime}-p^{\prime}+2$ is even.

If there exists $1 \leq k \leq p$ such that $\left[w_{k}\right]=\left[a_{j} w_{k}^{\prime}\right]$, then $w\left(\Gamma^{\prime}, v_{0}^{\prime}, \operatorname{ord}^{\prime}\right)=$ $\left\{w_{k}^{\prime} u^{\prime},\left[w_{1}\right],\left[w_{2}\right], \ldots,\left[w_{k-1}\right],\left[w_{k+1}\right], \ldots,\left[w_{p}\right]\right\}$. Hence, $p^{\prime}=p-1$ and $n-p=$ $\left(n^{\prime}+1\right)-\left(p^{\prime}+1\right)=n^{\prime}-p^{\prime}$ is even.

\section{The Degree Theorem}

Recall $\pi_{1}\left(\Gamma, v_{0}\right) \simeq F_{n}$.

We denote the valence of $v \in V(\Gamma)$ by $\left|v^{*}\right|$. 
5.1 Definition. The degree of $\left(\Gamma, v_{0}\right)$ is $2 n-\left|v_{0}^{*}\right|$. Equivalently, the degree of $\left(\Gamma, v_{0}\right)$ is $\sum_{v \in V(\Gamma)-\left\{v_{0}\right\}}\left(\left|v^{*}\right|-2\right)$.

To see the equivalence of the two definitions see [2, p. 636].

From the first definition of the degree of $\left(\Gamma, v_{0}\right)$ we see that when we collapse an edge of $\Gamma$ which is not incident with $v_{0}$ the degree is preserved, and, when we collapse an edge of $\Gamma$ which is incident with $v_{0}$ the degree decreases. Hence, graphs of degree at most $i$ expand a subcomplex $D_{i}$ of $\mathrm{SA}_{n}$. Hatcher and Vogtmann [2] proof the following.

5.2 Theorem. $D_{i}$ is $i$-dimensional and $(i-1)$-connected.

In particular, $D_{2}$ is a simply-connected 2-complex.

We define $\operatorname{ord} D_{i}$ for ordSA $\mathbb{A}_{g, p}$ as we define $D_{i}$ for $\mathrm{SA}_{n}$.

All the arguments of Hatcher and Vogtmann to proof [2, THEOREM 3.3] can be applied to $\operatorname{ord} \mathbb{A}_{g, p}$. In particular, what they call "canonical splitting" and "sliding in the $\epsilon$-cone" are combinations of splitting vertices and collapsing edges. We have the following.

5.3 Theorem. ord $D_{i}$ is $i$-dimensional and $(i-1)$-connected.

In particular, $\operatorname{ord} D_{2}$ is a simply-connected 2-complex.

\section{The action of $\mathcal{A} \mathcal{M}_{g, 1, p}$ on ord $\mathbb{A}_{n}$}

Recall that $\operatorname{Aut}\left(F_{n}\right)$ acts on $\mathbb{A}_{n}$ by "changing" the markings: for every $\varphi \in$ $\operatorname{Aut}\left(F_{n}\right)$ we define $\varphi \cdot\left(\Gamma, v_{0}, \phi\right)=\left(\Gamma, v_{0}, \varphi \circ \phi\right)$. This action restricts to $\mathrm{SA}_{n}$ and to $D_{2}$. The stabilizer of a vertex of $\mathrm{SA}_{n}$ by this action is a finite group which permutes some edges and invert some edge orientations. The quotient complex $\operatorname{Aut}\left(F_{n}\right) \backslash \mathrm{SA}_{n}$ is finite. See [1, Section 3], [2, Section 5]. Armstrong, Forrest and Vogtmann [1] apply a result of Brown [5] to $\operatorname{Aut}\left(F_{n}\right) \backslash D_{2}$ to compute a new presentation of $\operatorname{Aut}\left(F_{n}\right)$. Following this argument, we want to obtain a presentation of $\mathcal{A} \mathcal{M}_{g, 1, p}$.

We can define an action of $\mathcal{A \mathcal { M }}_{g, 1, p}$ on ord $\mathbb{A}_{g, p}$ by "changing" the markings: for every $\varphi \in \mathcal{A M}_{g, 1, p}$ we define $\varphi \cdot\left(\Gamma, v_{0}, \phi\right.$, ord $)=\left(\Gamma, v_{0}, \varphi \circ \phi\right.$, ord $)$. This action restricts to ordS $\mathbb{A}_{g, p}$ and to ord $D_{2}$. The stabilizer of a vertex of ordSA $\mathbb{A}_{g, p}$ by this action is trivial and the quotient complex $\mathcal{A M}_{g, 1, p} \backslash$ ordSA $\mathbb{A}_{g, p}$ is finite, but much bigger than $\operatorname{Aut}\left(F_{n}\right) \backslash \mathrm{SA}_{n}$. By Theorem [5.3, ord $D_{2}$ is simply-connected. Hence, $\mathcal{A} \mathcal{M}_{g, 1, p}$ is isomorphic to the fundamental group of $\mathcal{A} \mathcal{M}_{g, 1, p} \backslash \operatorname{ord} D_{2}$. In the next section we give an algorithm which computes a presentation of the fundamental group of $\mathcal{A M}_{g, 1, p} \backslash$ ord $D_{2}$. 


\section{The algorithm}

Recall $n=2 g+p$.

Vertices of ordSA $\mathbb{A}_{g, p}$ are represented by 4 -tuples $\left(\Gamma, v_{0}, \phi\right.$, ord $)$ such that $w\left(\Gamma, v_{0}\right.$, ord $)$ has $p$ conjugacy classes. Recall that $\varphi \in \mathcal{A M}_{g, 1, p}$ acts on ordSA $\mathbb{A}_{g, p}$ by "changing" the marking, that is, $\varphi \cdot\left(\Gamma, v_{0}, \phi\right.$, ord $)=\left(\Gamma, v_{0}, \varphi \circ \phi\right.$, ord $)$. Hence,

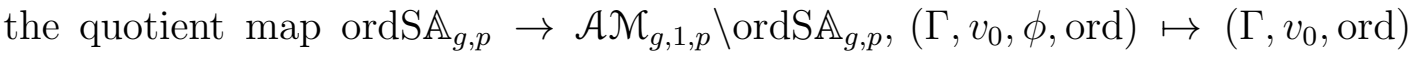
"forgets" the marking. We can represent vertices of $\mathcal{A} \mathcal{M}_{g, 1, p} \backslash$ ordSA $\mathbb{A}_{g, p}$ by 3 -tuples $\left(\Gamma, v_{0}\right.$, ord $)$ such that $w\left(\Gamma, v_{0}\right.$, ord $)$ has $p$ conjugacy classes. Vertices of the subcomplex $\mathcal{A M}_{g, 1, p} \backslash$ ord $D_{2}$ can be represented by 3 -tuples $\left(\Gamma, v_{0}\right.$, ord $)$ such that $\left(\Gamma, v_{0}\right)$ has degree at most 2.

We want to compute a presentation for the fundamental group of complex $\mathcal{A} \mathcal{M}_{g, 1, p} \backslash \operatorname{ord} D_{2}$. Recall that the degree of $\left(\Gamma, v_{0}\right)$ can be defined as $\sum_{v \in V(\Gamma)-\left\{v_{0}\right\}}\left(\left|v^{*}\right|-2\right)$. Hence, if $\left(\Gamma, v_{0}\right)$ has degree 2 then $\Gamma$ has at most three vertices: $v_{0}$ and two more vertices of valence 3 .

Let $\mathcal{L}$ be a list of vertices $\left(\Gamma, v_{0}\right.$, ord) of $\mathcal{A M}_{g, 1, p} \backslash$ ord $D_{2}$ such that $\Gamma$ has 3 vertices.

Let $z=\left(\Gamma, v_{0}\right.$, ord $)$ be an element of $\mathcal{L}$. Suppose $E(\Gamma)=\left\{e_{1}, e_{2}, \ldots e_{k}\right\}$.

We construct a tree $T(z)$ as follows. There exists a vertex $z$ of $T(z)$. Let $e_{i}$ be an edge of $\Gamma$ which can be collapsed, that is, $e_{i}$ is incident to two different vertices. When we collapse $e_{i}$ we have a quotient 3 -tuple $z^{i}=\left(\Gamma^{i}, v_{0}^{i}\right.$, ord $\left.{ }^{i}\right)$. There exists a vertex $z^{i}$ of $T(z)$ and an edge $z e_{i}$ of $T(z)$ from $z$ to $z^{i}$. We identify edges of $z^{i}$ with edges of $z$. Let $e_{j}$ be an edge of $\Gamma^{i}$ which can be collapsed. When we collapse $e_{j}$ in $\Gamma^{i}$ we have a quotient 3 -tuple $z^{(i, j)}=\left(\Gamma^{(i, j)}, v_{0}^{(i, j)}\right.$, ord $\left.{ }^{(i, j)}\right)$. There exists a vertex $z^{(i, j)}$ of $T(z)$ and an edge $z e_{i} e_{j}$ from $z^{i}$ to $z^{(i, j)}$. We repeat this process for every edge which can be collapsed.

Our generating set for the fundamental group of $\mathcal{A} \mathcal{M}_{g, 1, p} \backslash \operatorname{ord} D_{2}$ is the set of edges of $T(z)$, where $z$ ranges over $\mathcal{L}$.

The group $\operatorname{Sym}_{k} \times C_{2}^{\times k}$ acts on $E(\Gamma) \cup \bar{E}(\Gamma)$ by permuting edges $\left(C_{2}^{\times k}\right.$ is the Cartesian product of $k$ copies of the cyclic group of order 2). Hence, $\operatorname{Sym}_{k} \times C_{2}^{\times k}$ acts on the set of 3 -tuples $\left(\Gamma, v_{0}\right.$, ord) by permuting edges and inverting edge orientations. Two 3-tuples $\left(\Gamma, v_{0}\right.$, ord $)$ and $\left(\Gamma^{\prime}, v_{0}^{\prime}, \operatorname{ord}^{\prime}\right)$ represent the same vertex of $\mathcal{A} \mathcal{M}_{g, 1, p} \backslash$ ordSA $\mathbb{A}_{g, p}$ if and only if they are in the same orbit by the action of $\operatorname{Sym}_{k} \times C_{2}^{\times k}$. Since every vertex of $T(z)$ is a 3 -tuple $\left(\Gamma, v_{0}\right.$, ord $)$, we see that $\operatorname{Sym}_{k} \times C_{2}^{\times k}$ acts on $T(z)$. We can identify $\left(\operatorname{Sym}_{k} \times C_{2}^{\times k}\right) \backslash T(z)$ with the 1-skeleton of a subcomplex of $\mathcal{A} \mathcal{M}_{g, 1, p} \backslash \operatorname{ord} D_{2}$. We can identify

$$
\left(\operatorname{Sym}_{k} \times C_{2}^{\times k}\right) \backslash\left(\bigcup_{z \in \mathcal{L}} T(z)\right)
$$

with the 1-skeleton of a subcomplex of $\mathcal{A M}_{g, 1, p} \backslash \operatorname{ord} D_{2}$.

We attach some 2-cells to $\left(\operatorname{Sym}_{k} \times C_{2}^{\times k}\right) \backslash\left(\bigcup_{z \in \mathcal{L}} T(z)\right)$. If there exists the generator $z e_{i} e_{j}$, we attach a 2 -cell though the egde-path $z e_{i}, z e_{i} e_{j}, \overline{z e_{j} e_{i}}, \overline{z e_{j}}$. With 
these attached 2-cells, the 2-complex $\left(\operatorname{Sym}_{k} \times C_{2}^{\times k}\right) \backslash\left(\bigcup_{z \in \mathcal{L}} T(z)\right)$ is homeomorphic to $\mathcal{A} \mathcal{M}_{g, 1, p} \backslash$ ord $D_{2}$. We fix a maximal subtree of $\left(\operatorname{Sym}_{k} \times C_{2}^{\times k}\right) \backslash\left(\bigcup_{z \in \mathcal{L}} T(z)\right)$.

Our presentation for the fundamental group of $\mathcal{A} \mathcal{M}_{g, 1, p} \backslash \operatorname{ord} D_{2}$ has three types of relations:

(a). $z e_{i}=1, z e_{i^{\prime}} e_{j^{\prime}}=1$ if the edges $z e_{i}, z e_{i^{\prime}} e_{j^{\prime}}$ are in our maximal subtree.

(b). $z_{1} e_{i} e_{j}=z_{2} e_{i^{\prime}} e_{j^{\prime}}$ if the generator $z_{1} e_{i} e_{j}$ exists and $g \cdot z_{2}^{i^{\prime}}=z_{1}^{i}$ for some $g \in \operatorname{Sym}_{k} \times C_{2}^{\times k}$ such that either $g \cdot e_{j^{\prime}}=e_{j}$ or $g \cdot e_{j^{\prime}}=\bar{e}_{j}$.

(c). $z e_{i} \cdot z e_{i} e_{j}=z e_{j} \cdot z e_{j} e_{i}$ if there exists the generator $z e_{i} e_{j}$.

We illustrate the algorithm with two easy examples. The main difficulty of the algorithm is to find $\mathcal{L}$. Once $\mathcal{L}$ is known our, it is straightforward to apply the algorithm. Example 7.2 shows that the algorithm can be applied in "pieces", each piece corresponding to an element of $\mathcal{L}$.

7.1 Example. We take $(g, p)=(1,0)$. The list $\mathcal{L}$ has a single element. We can represent the element of $\mathcal{L}$ by

$$
z=\left(V(\Gamma), E(\Gamma), V^{*}(\Gamma), \text { ord }\right)=\left(\left\{v_{0}, v_{1}, v_{2}\right\},\left\{e_{1}, e_{2}, e_{3}, e_{4}\right\},\left\{v_{0}^{*}, v_{1}^{*}, v_{2}^{*}\right\}, \text { ord }\right),
$$

where $\operatorname{ord}\left(v_{0}^{*}\right)=\left(e_{1}, e_{2}\right), \operatorname{ord}\left(v_{1}^{*}\right)=\left(\bar{e}_{1}, e_{3}, e_{4}\right)$ and $\operatorname{ord}\left(v_{2}^{*}\right)=\left(\bar{e}_{2}, \bar{e}_{3}, \bar{e}_{4}\right)$. To simplify the notation we put

$$
z=\operatorname{ord}\left(v_{0}^{*}\right) ; \operatorname{ord}\left(v_{1}^{*}\right), \operatorname{ord}\left(v_{2}^{*}\right)=\left(e_{1}, e_{2}\right) ;\left(\bar{e}_{1}, e_{3}, e_{4}\right),\left(\bar{e}_{2}, \bar{e}_{3}, \bar{e}_{4}\right) .
$$

We can collapse all 4 edges of $z$ and we have

$$
\begin{aligned}
& z^{1}=\left(e_{3}, e_{4}, e_{2}\right) ;\left(\bar{e}_{2}, \bar{e}_{3}, \bar{e}_{4}\right), \\
& z^{2}=\left(e_{1}, \bar{e}_{3}, \bar{e}_{4}\right) ;\left(\bar{e}_{1}, e_{3}, e_{4}\right), \\
& z^{3}=\left(e_{1}, e_{2}\right) ;\left(\bar{e}_{1}, \bar{e}_{4}, \bar{e}_{2}, e_{4}\right), \\
& z^{4}=\left(e_{1}, e_{2}\right) ;\left(\bar{e}_{1}, e_{3}, \bar{e}_{2}, \bar{e}_{3}\right) .
\end{aligned}
$$

We see $z^{1}=g^{2,1} \cdot z^{2}, z^{3}=g^{4,3} \cdot z^{4}$, where $g^{2,1}, g^{4,3} \in \operatorname{Sym}_{4} \times C_{2}^{\times 4}$ and

$$
g^{2,1}=\left\{\begin{aligned}
e_{1} & \mapsto e_{3}, \\
e_{3} & \mapsto \bar{e}_{4}, \\
e_{4} & \mapsto \bar{e}_{2},
\end{aligned} \quad \text { and } \quad g^{4,3}=\left\{\begin{aligned}
e_{1} & \mapsto e_{1}, \\
e_{2} & \mapsto e_{2}, \\
e_{3} & \mapsto \bar{e}_{4} .
\end{aligned}\right.\right.
$$

We can collapse some edges of $z^{1}$ and $z^{3}$ and we have

$$
\begin{array}{ll}
z^{(1,3)}=\left(\bar{e}_{4}, \bar{e}_{2}, e_{4}, e_{2}\right), \\
z^{(1,4)}=\left(e_{3}, \bar{e}_{2}, \bar{e}_{3}, e_{2}\right), \\
z^{(1,2)}=\left(e_{3}, e_{4}, \bar{e}_{3}, \bar{e}_{4}\right) ;
\end{array} \quad \text { and } \quad \begin{array}{ll}
z^{(3,1)} & =\left(\bar{e}_{4}, \bar{e}_{2}, e_{4}, e_{2}\right),
\end{array}
$$


We see $z^{(1,2)}, z^{(1,3)}, z^{(1,4)}, z^{(3,1)}$ and $z^{(3,2)}$ are in the same orbit by $\mathrm{Sym}_{4} \times C_{2}^{\times 4}$. Hence, they represent the same vertex of $\left(\mathrm{Sym}_{4} \times C_{2}^{\times 4}\right) \backslash T(z)$.

We take the maximal subtree of $\left(\operatorname{Sym}_{4} \times C_{2}^{\times 4}\right) \backslash T(z)$ with edges $z e_{1}, z e_{3}$ and $z e_{1} e_{2}$. Then $\mathcal{A M}_{1,0}$ has presentation with generators:

$$
\begin{aligned}
& z e_{1}, z e_{2}, z e_{3}, z e_{4}, \\
& z e_{1} e_{3}, z e_{1} e_{4}, z e_{1} e_{2}, \\
& z e_{2} e_{1}, z e_{2} e_{3}, z e_{2} e_{4}, \\
& z e_{3} e_{1}, z e_{3} e_{2}, \\
& z e_{4} e_{1}, z e_{4} e_{2} ;
\end{aligned}
$$

and relations:

$$
\begin{aligned}
& z e_{1}=1, z e_{3}=1, z e_{1} e_{2}=1, \\
& z e_{2} e_{1}=z e_{1} e_{3}, z e_{2} e_{3}=z e_{1} e_{4}, z e_{2} e_{4}=z e_{1} e_{2}, z e_{4} e_{1}=z e_{3} e_{1}, z e_{4} e_{2}=z e_{3} e_{2}, \\
& z e_{1} \cdot z e_{1} e_{2}=z e_{2} \cdot z e_{2} e_{1}, z e_{1} \cdot z e_{1} e_{3}=z e_{3} \cdot z e_{3} e_{1}, z e_{1} \cdot z e_{1} e_{4}=z e_{4} \cdot z e_{4} e_{1}, \\
& \quad z e_{2} \cdot z e_{2} e_{3}=z e_{3} \cdot z e_{3} e_{2}, z e_{2} \cdot z e_{2} e_{4}=z e_{4} \cdot z e_{4} e_{2} .
\end{aligned}
$$

An easy simplification shows $\mathcal{A M}_{1,1,0}=\left\langle z e_{2}, z e_{4} \mid z e_{2} \cdot z e_{2}=z e_{4} \cdot z e_{2} \cdot z e_{4}\right\rangle$.

7.2 Example. We take $(g, p)=(0,3)$. The list $\mathcal{L}$ is

$$
\begin{aligned}
& z_{1}=\left(e_{1}, e_{2}, e_{3}, e_{4}\right) ;\left(\bar{e}_{1}, e_{5}, \bar{e}_{2}\right),\left(\bar{e}_{3}, \bar{e}_{5}, \bar{e}_{4}\right), \\
& z_{2}=\left(e_{1}, e_{2}, e_{3}, e_{4}\right) ;\left(\bar{e}_{1}, \bar{e}_{4}, e_{5}\right),\left(\bar{e}_{2}, \bar{e}_{5}, \bar{e}_{3}\right), \\
& z_{3}=\left(e_{1}, \bar{e}_{1}, e_{2}, e_{3}\right) ;\left(\bar{e}_{2}, e_{4}, e_{5}\right),\left(\bar{e}_{3}, \bar{e}_{5}, \bar{e}_{4}\right), \\
& z_{4}=\left(e_{1}, e_{2}, \bar{e}_{2}, e_{3}\right) ;\left(\bar{e}_{1}, e_{4}, e_{5}\right),\left(\bar{e}_{3}, \bar{e}_{5}, \bar{e}_{4}\right), \\
& z_{5}=\left(e_{1}, e_{2}, e_{3}, \bar{e}_{3}\right) ;\left(\bar{e}_{1}, e_{4}, e_{5}\right),\left(\bar{e}_{2}, \bar{e}_{5}, \bar{e}_{4}\right), \\
& z_{6}=\left(e_{1}, e_{2}, e_{3}, \bar{e}_{1}\right) ;\left(\bar{e}_{2}, e_{4}, e_{5}\right),\left(\bar{e}_{3}, \bar{e}_{5}, \bar{e}_{4}\right) .
\end{aligned}
$$

For $z_{1}$ we have

$$
\begin{aligned}
z_{1}= & \left(e_{1}, e_{2}, e_{3}, e_{4}\right) ;\left(\bar{e}_{1}, e_{5}, \bar{e}_{2}\right),\left(\bar{e}_{3}, \bar{e}_{5}, \bar{e}_{4}\right), \\
z_{1}^{1} & =\left(e_{5}, \bar{e}_{2}, e_{2}, e_{3}, e_{4}\right) ;\left(\bar{e}_{3}, \bar{e}_{5}, \bar{e}_{4}\right), \\
z_{1}^{2} & =\left(e_{1}, \bar{e}_{1}, e_{5}, e_{3}, e_{4}\right) ;\left(\bar{e}_{3}, \bar{e}_{5}, \bar{e}_{4}\right), \\
z_{1}^{3} & =\left(e_{1}, e_{2}, \bar{e}_{5}, \bar{e}_{4}, e_{4}\right) ;\left(\bar{e}_{1}, e_{5}, \bar{e}_{2}\right) \\
z_{1}^{4} & =\left(e_{1}, e_{2}, e_{3}, \bar{e}_{3}, \bar{e}_{5}\right) ;\left(\bar{e}_{1}, e_{5}, \bar{e}_{2}\right) \\
z_{1}^{5} & =\left(e_{1}, e_{2}, e_{3}, e_{4}\right) ;\left(\bar{e}_{1}, \bar{e}_{4}, \bar{e}_{3}, \bar{e}_{2}\right) .
\end{aligned}
$$

Generators for $z_{1}$ are:

$$
\begin{aligned}
& z_{1} e_{1}, z_{1} e_{2}, z_{1} e_{3}, z_{1} e_{4}, z_{1} e_{5}, \\
& z_{1} e_{1} e_{5}, z_{1} e_{1} e_{3}, z_{1} e_{1} e_{4}, \\
& z_{1} e_{2} e_{5}, z_{1} e_{2} e_{3}, z_{1} e_{2} e_{4}, \\
& z_{1} e_{3} e_{1}, z_{1} e_{3} e_{2}, z_{1} e_{3} e_{5}, \\
& z_{1} e_{4} e_{1}, z_{1} e_{4} e_{2}, z_{1} e_{4} e_{5}, \\
& z_{1} e_{5} e_{1}, z_{1} e_{5} e_{2}, z_{1} e_{5} e_{3}, z_{1} e_{5} e_{4} .
\end{aligned}
$$


We have

$$
\begin{aligned}
& z_{1}^{(1,5)}=\left(\bar{e}_{4}, \bar{e}_{3}, \bar{e}_{2}, e_{2}, e_{3}, e_{4}\right), \\
& z_{1}^{(1,3)}=\left(e_{5}, \bar{e}_{2}, e_{2}, \bar{e}_{5}, \bar{e}_{4}, e_{4}\right), \\
& z_{1}^{(1,4)}=\left(e_{5}, \bar{e}_{2}, e_{2}, e_{3}, \bar{e}_{3}, \bar{e}_{5}\right), \\
& z_{1}^{(2,5)}=\left(e_{1}, \bar{e}_{1}, \bar{e}_{4}, \bar{e}_{3}, e_{3}, e_{4}\right), \\
& z_{1}^{(2,3)}=\left(e_{1}, \bar{e}_{1}, e_{5}, \bar{e}_{5}, \bar{e}_{4}, e_{4}\right), \\
& z_{1}^{(2,4)}=\left(e_{1}, \bar{e}_{1}, e_{5}, e_{3}, \bar{e}_{3}, \bar{e}_{5}\right), \\
& z_{1}^{(3,5)}=\left(e_{1}, e_{2}, \bar{e}_{2}, \bar{e}_{1}, \bar{e}_{4}, e_{4}\right), \\
& z_{1}^{(4,5)}=\left(e_{1}, e_{2}, e_{3}, \bar{e}_{3}, \bar{e}_{2}, \bar{e}_{1}\right) .
\end{aligned}
$$

We see that $z_{1}^{(2,4)}=g \cdot z_{1}^{(2,5)}, z_{1}^{(3,5)}=g^{\prime} \cdot z_{1}^{(1,3)}, z_{1}^{(4,5)}=g^{\prime \prime} \cdot z_{1}^{(1,5)}$ for some $g, g^{\prime}, g^{\prime \prime} \in \mathrm{Sym}_{5} \times C_{2}^{\times 5}$.

Relations for $z_{1}$ are:

$$
\begin{aligned}
& z_{1} e_{1}=1, z_{1} e_{2}=1, z_{1} e_{3}=1, z_{1} e_{4}=1, z_{1} e_{5}=1, \\
& z_{1} e_{1} e_{5}=1, z_{1} e_{1} e_{3}=1, z_{1} e_{1} e_{4}=1, z_{1} e_{2} e_{5}=1, z_{1} e_{2} e_{3}=1, \\
& z_{1} e_{1} \cdot z_{1} e_{1} e_{5}=z_{1} e_{5} \cdot z_{1} e_{5} e_{1}, z_{1} e_{1} \cdot z_{1} e_{1} e_{3}=z_{1} e_{3} \cdot z_{1} e_{3} e_{1}, \\
& \quad z_{1} e_{1} \cdot z_{1} e_{1} e_{4}=z_{1} e_{4} \cdot z_{1} e_{4} e_{1}, \\
& z_{1} e_{2} \cdot z_{1} e_{2} e_{5}=z_{1} e_{5} \cdot z_{1} e_{5} e_{2}, z_{1} e_{2} \cdot z_{1} e_{2} e_{3}=z_{1} e_{3} \cdot z_{1} e_{3} e_{2}, \\
& \quad z_{1} e_{2} \cdot z_{1} e_{2} e_{4}=z_{1} e_{4} \cdot z_{1} e_{4} e_{2}, \\
& z_{1} e_{3} \cdot z_{1} e_{3} e_{5}=z_{1} e_{5} \cdot z_{1} e_{5} e_{3}, \\
& z_{1} e_{4} \cdot z_{1} e_{4} e_{5}=z_{1} e_{5} \cdot z_{1} e_{5} e_{4} .
\end{aligned}
$$

An easy simplification shows that for $z_{1}$ generators are $z_{1} e_{2} e_{4}, z_{1} e_{3} e_{5}, z_{1} e_{4} e_{5}$ and for $z_{1}$ there are no relations.

From $z_{2}$ we have

$$
\begin{aligned}
z_{2}= & \left(e_{1}, e_{2}, e_{3}, e_{4}\right) ;\left(\bar{e}_{1}, \bar{e}_{4}, e_{5}\right),\left(\bar{e}_{2}, \bar{e}_{5}, \bar{e}_{3}\right), \\
z_{2}^{1} & =\left(\bar{e}_{4}, e_{5}, e_{2}, e_{3}, e_{4}\right) ;\left(\bar{e}_{2}, \bar{e}_{5}, \bar{e}_{3}\right), \\
z_{2}^{2} & =\left(e_{1}, \bar{e}_{5}, \bar{e}_{3}, e_{3}, e_{4}\right) ;\left(\bar{e}_{1}, \bar{e}_{4}, e_{5}\right), \\
z_{2}^{3} & =\left(e_{1}, e_{2}, \bar{e}_{2}, \bar{e}_{5}, e_{4}\right) ;\left(\bar{e}_{1}, \bar{e}_{4}, e_{5}\right), \\
z_{2}^{4} & =\left(e_{1}, e_{2}, e_{3}, e_{5}, \bar{e}_{1}\right) ;\left(\bar{e}_{2}, \bar{e}_{5}, \bar{e}_{3}\right), \\
z_{2}^{5} & =\left(e_{1}, e_{2}, e_{3}, e_{4}\right) ;\left(\bar{e}_{1}, \bar{e}_{4}, \bar{e}_{3}, \bar{e}_{2}\right) .
\end{aligned}
$$


Generators for $z_{2}$ are:

$$
\begin{aligned}
& z_{2} e_{1}, z_{2} e_{2}, z_{2} e_{3}, z_{2} e_{4}, z_{2} e_{5}, \\
& z_{2} e_{1} e_{5}, z_{2} e_{1} e_{2}, z_{2} e_{1} e_{3}, \\
& z_{2} e_{2} e_{1}, z_{2} e_{2} e_{5}, z_{2} e_{2} e_{4}, \\
& z_{2} e_{3} e_{1}, z_{2} e_{3} e_{5}, z_{2} e_{3} e_{4}, \\
& z_{2} e_{4} e_{2}, z_{2} e_{4} e_{3}, z_{2} e_{4} e_{5}, \\
& z_{2} e_{5} e_{1}, z_{2} e_{5} e_{2}, z_{2} e_{5} e_{3}, z_{2} e_{5} e_{4} .
\end{aligned}
$$

We see $z_{1}^{4}=g_{2,1}^{2,4} \cdot z_{2}^{2}, z_{1}^{1}=g_{2,1}^{3,1} \cdot z_{2}^{3}, z_{2}^{1}=g_{2,2}^{4,1} \cdot z_{2}^{4}, z_{1}^{5}=g_{2,1}^{5,5} \cdot z_{2}^{5}$, where

$$
\begin{aligned}
& g_{2,1}^{2,4}=\left\{\begin{array}{rl}
e_{1} & \mapsto e_{1}, \\
e_{3} & \mapsto \bar{e}_{3}, \\
e_{4} & \mapsto \bar{e}_{5}, \\
e_{5} & \mapsto \bar{e}_{2} ;
\end{array} \quad g_{2,1}^{3,1}=\left\{\begin{array}{rl}
e_{1} & \mapsto e_{5}, \\
e_{2} & \mapsto \bar{e}_{2}, \\
e_{4} & \mapsto e_{4}, \\
e_{5} & \mapsto \bar{e}_{3} ;
\end{array} \quad g_{2,2}^{4,1}=\left\{\begin{array}{l}
e_{1} \mapsto \bar{e}_{4}, \\
e_{2} \mapsto e_{5}, \\
e_{3} \mapsto e_{2}, \\
e_{5} \mapsto e_{3} ;
\end{array}\right.\right.\right. \\
& g_{2,1}^{5,5}=\left\{\begin{aligned}
e_{1} & \mapsto e_{1}, \\
e_{2} & \mapsto e_{2}, \\
e_{3} & \mapsto e_{3}, \\
e_{4} & \mapsto e_{4}
\end{aligned}\right.
\end{aligned}
$$

Relations for $z_{2}$ are:

$$
\begin{aligned}
& z_{2} e_{1}=1, z_{2} e_{2}=1, \\
& z_{2} e_{2} e_{1}=z_{1} e_{4} e_{1}, z_{2} e_{2} e_{5}=z_{1} e_{4} e_{2}, z_{2} e_{2} e_{4}=z_{1} e_{4} e_{5}, \\
& z_{2} e_{3} e_{1}=z_{1} e_{1} e_{5}, z_{2} e_{3} e_{5}=z_{1} e_{1} e_{3}, z_{2} e_{3} e_{4}=z_{1} e_{1} e_{4}, \\
& z_{2} e_{4} e_{2}=z_{2} e_{1} e_{5}, z_{2} e_{4} e_{3}=z_{2} e_{1} e_{2}, z_{2} e_{4} e_{5}=z_{2} e_{1} e_{3}, \\
& z_{2} e_{5} e_{1}=z_{1} e_{5} e_{1}, z_{2} e_{5} e_{2}=z_{1} e_{5} e_{2}, z_{2} e_{5} e_{3}=z_{1} e_{5} e_{3}, z_{2} e_{5} e_{4}=z_{1} e_{5} e_{4}, \\
& z_{2} e_{1} \cdot z_{2} e_{1} e_{5}=z_{2} e_{5} \cdot z_{2} e_{5} e_{1}, z_{2} e_{1} \cdot z_{2} e_{1} e_{2}=z_{2} e_{2} \cdot z_{2} e_{2} e_{1}, \\
& \quad z_{2} e_{1} \cdot z_{2} e_{1} e_{3}=z_{2} e_{3} \cdot z_{2} e_{3} e_{1}, \\
& z_{2} e_{2} \cdot z_{2} e_{2} e_{5}=z_{2} e_{5} \cdot z_{2} e_{5} e_{2}, z_{2} e_{2} \cdot z_{2} e_{2} e_{4}=z_{2} e_{4} \cdot z_{2} e_{4} e_{2}, \\
& z_{2} e_{3} \cdot z_{2} e_{3} e_{5}=z_{2} e_{5} \cdot z_{2} e_{5} e_{3}, z_{2} e_{3} \cdot z_{2} e_{3} e_{4}=z_{2} e_{4} \cdot z_{2} e_{4} e_{3}, \\
& z_{2} e_{4} \cdot z_{2} e_{4} e_{5}=z_{2} e_{5} \cdot z_{2} e_{5} e_{4} .
\end{aligned}
$$

An easy simplification shows no new generators are needed, the relations $z_{1} e_{4} e_{5}=z_{1} e_{2} e_{4} \cdot z_{1} e_{3} e_{5} \cdot z_{1} e_{2} e_{4}, z_{1} e_{4} e_{5} \cdot z_{1} e_{3} e_{5}=z_{1} e_{2} e_{4} \cdot z_{1} e_{4} e_{5}$ are needed.

From $z_{3}$ we have

$$
\begin{aligned}
z_{3}= & \left(e_{1}, \bar{e}_{1}, e_{2}, e_{3}\right) ;\left(\bar{e}_{2}, e_{4}, e_{5}\right),\left(\bar{e}_{3}, \bar{e}_{5}, \bar{e}_{4}\right), \\
z_{3}^{2} & =\left(e_{1}, \bar{e}_{1}, e_{4}, e_{5}, e_{3}\right) ;\left(\bar{e}_{3}, \bar{e}_{5}, \bar{e}_{4}\right), \\
z_{3}^{3} & =\left(e_{1}, \bar{e}_{1}, e_{2}, \bar{e}_{5}, \bar{e}_{4}\right) ;\left(\bar{e}_{2}, e_{4}, e_{5}\right), \\
z_{3}^{4} & =\left(e_{1}, \bar{e}_{1}, e_{2}, e_{3}\right) ;\left(\bar{e}_{2}, \bar{e}_{3}, \bar{e}_{5}, e_{5}\right), \\
z_{3}^{5} & =\left(e_{1}, \bar{e}_{1}, e_{2}, e_{3}\right) ;\left(\bar{e}_{2}, e_{4}, \bar{e}_{4}, \bar{e}_{3}\right) .
\end{aligned}
$$


Generators for $z_{3}$ are:

$$
\begin{aligned}
& z_{3} e_{2}, z_{3} e_{3}, z_{3} e_{4}, z_{3} e_{5}, \\
& z_{3} e_{2} e_{4}, z_{3} e_{2} e_{5}, z_{3} e_{2} e_{3}, \\
& z_{3} e_{3} e_{2}, z_{3} e_{3} e_{5}, z_{3} e_{3} e_{4}, \\
& z_{3} e_{4} e_{2}, z_{3} e_{4} e_{3}, \\
& z_{3} e_{5} e_{2}, z_{3} e_{5} e_{3} .
\end{aligned}
$$

We see $z_{1}^{2}=g_{3,1}^{2,2} \cdot z_{3}^{2}, z_{1}^{2}=g_{3,1}^{3,2} \cdot z_{3}^{3}$, where

$$
g_{3,1}^{2,2}=\left\{\begin{array}{rl}
e_{1} & \mapsto e_{1}, \\
e_{3} & \mapsto e_{4}, \\
e_{4} & \mapsto e_{5}, \\
e_{5} & \mapsto e_{3} ;
\end{array} \quad g_{3,1}^{3,2}=\left\{\begin{aligned}
e_{1} & \mapsto e_{1}, \\
e_{2} & \mapsto e_{5}, \\
e_{4} & \mapsto \bar{e}_{4}, \\
e_{5} & \mapsto \bar{e}_{3} .
\end{aligned}\right.\right.
$$

Relations for $z_{3}$ are:

$$
\begin{aligned}
& z_{3} e_{2}, z_{3} e_{4}=1, z_{3} e_{5}=1, \\
& z_{3} e_{2} e_{4}=z_{1} e_{2} e_{5}, z_{3} e_{2} e_{5}=z_{1} e_{2} e_{3}, z_{3} e_{2} e_{3}=z_{1} e_{2} e_{4}, \\
& z_{3} e_{3} e_{2}=z_{1} e_{2} e_{5}, z_{3} e_{3} e_{5}=z_{1} e_{2} e_{3}, z_{3} e_{3} e_{4}=z_{1} e_{2} e_{4}, \\
& z_{3} e_{2} \cdot z_{3} e_{2} e_{4}=z_{3} e_{4} \cdot z_{3} e_{4} e_{2}, z_{3} e_{2} \cdot z_{3} e_{2} e_{5}=z_{3} e_{5} \cdot z_{3} e_{5} e_{2}, \\
& \quad z_{3} e_{2} \cdot z_{3} e_{2} e_{3}=z_{3} e_{3} \cdot z_{3} e_{3} e_{2}, \\
& z_{3} e_{3} \cdot z_{3} e_{3} e_{5}=z_{3} e_{5} \cdot z_{3} e_{5} e_{3}, z_{3} e_{3} \cdot z_{3} e_{3} e_{4}=z_{3} e_{4} \cdot z_{3} e_{4} e_{3} .
\end{aligned}
$$

An easy simplification shows that neither new generators nor new relations are needed.

From $z_{4}$ we have

$$
\begin{aligned}
z_{4}= & \left(e_{1}, e_{2}, \bar{e}_{2}, e_{3}\right) ;\left(\bar{e}_{1}, e_{4}, e_{5}\right),\left(\bar{e}_{3}, \bar{e}_{5}, \bar{e}_{4}\right), \\
z_{4}^{1} & =\left(e_{4}, e_{5}, e_{2}, \bar{e}_{2}, e_{3}\right) ;\left(\bar{e}_{3}, \bar{e}_{5}, \bar{e}_{4}\right), \\
z_{4}^{3} & =\left(e_{1}, e_{2}, \bar{e}_{2}, \bar{e}_{5}, \bar{e}_{4}\right) ;\left(\bar{e}_{1}, e_{4}, e_{5}\right), \\
z_{4}^{4} & =\left(e_{1}, e_{2}, \bar{e}_{2}, e_{3}\right) ;\left(\bar{e}_{1}, \bar{e}_{3}, \bar{e}_{5}, e_{5}\right), \\
z_{4}^{5} & =\left(e_{1}, e_{2}, \bar{e}_{2}, e_{3}\right) ;\left(\bar{e}_{1}, e_{4}, \bar{e}_{4}, \bar{e}_{3}\right) .
\end{aligned}
$$

Generators for $z_{4}$ are:

$$
\begin{aligned}
& z_{4} e_{1}, z_{4} e_{3}, z_{4} e_{4}, z_{4} e_{5}, \\
& z_{4} e_{1} e_{4}, z_{4} e_{1} e_{5}, z_{4} e_{1} e_{3}, \\
& z_{4} e_{3} e_{1}, z_{4} e_{3} e_{5}, z_{4} e_{3} e_{4}, \\
& z_{4} e_{4} e_{1}, z_{4} e_{4} e_{3}, \\
& z_{4} e_{5} e_{1}, z_{4} e_{5} e_{3} .
\end{aligned}
$$


We see $z_{1}^{4}=g_{4,1}^{1,4} \cdot z_{4}^{1}, z_{1}^{1}=g_{4,1}^{3,1} \cdot z_{4}^{3}$, where

$$
g_{4,1}^{1,4}=\left\{\begin{array}{rl}
e_{2} & \mapsto e_{3}, \\
e_{3} & \mapsto \bar{e}_{5}, \\
e_{4} & \mapsto e_{1}, \\
e_{5} & \mapsto e_{2} ;
\end{array} \quad g_{4,1}^{3,1}=\left\{\begin{aligned}
e_{1} & \mapsto e_{5}, \\
e_{2} & \mapsto e_{2}, \\
e_{4} & \mapsto \bar{e}_{4}, \\
e_{5} & \mapsto \bar{e}_{3}
\end{aligned}\right.\right.
$$

Relations for $z_{4}$ are:

$$
\begin{aligned}
& z_{4} e_{4}=1, z_{4} e_{4}=1, z_{4} e_{5}=1, \\
& z_{4} e_{1} e_{4}=z_{1} e_{4} e_{1}, z_{4} e_{1} e_{5}=z_{1} e_{4} e_{2}, z_{4} e_{1} e_{3}=z_{1} e_{4} e_{5}, \\
& z_{4} e_{3} e_{1}=z_{1} e_{1} e_{5}, z_{4} e_{3} e_{5}=z_{1} e_{1} e_{3}, z_{4} e_{3} e_{4}=z_{1} e_{1} e_{4}, \\
& z_{4} e_{1} \cdot z_{4} e_{1} e_{4}=z_{4} e_{4} \cdot z_{4} e_{4} e_{1}, z_{4} e_{1} \cdot z_{4} e_{1} e_{5}=z_{4} e_{5} \cdot z_{4} e_{5} e_{1}, \\
& \quad z_{4} e_{1} \cdot z_{4} e_{1} e_{3}=z_{4} e_{3} \cdot z_{4} e_{3} e_{1}, \\
& z_{4} e_{3} \cdot z_{4} e_{3} e_{5}=z_{4} e_{5} \cdot z_{4} e_{5} e_{3}, z_{4} e_{3} \cdot z_{4} e_{3} e_{4}=z_{4} e_{4} \cdot z_{4} e_{4} e_{3} .
\end{aligned}
$$

An easy simplification shows that neither new generators nor new relations are needed.

From $z_{5}$ we have

$$
\begin{aligned}
z_{5}= & \left(e_{1}, e_{2}, e_{3}, \bar{e}_{3}\right) ;\left(\bar{e}_{1}, e_{4}, e_{5}\right),\left(\bar{e}_{2}, \bar{e}_{5}, \bar{e}_{4}\right), \\
z_{5}^{1} & =\left(e_{4}, e_{5}, e_{2}, e_{3}, \bar{e}_{3}\right) ;\left(\bar{e}_{2}, \bar{e}_{5}, \bar{e}_{4}\right), \\
z_{5}^{2} & =\left(e_{1}, \bar{e}_{5}, \bar{e}_{4}, e_{3}, \bar{e}_{3}\right) ;\left(\bar{e}_{1}, e_{4}, e_{5}\right), \\
z_{5}^{4} & =\left(e_{1}, e_{2}, e_{3}, \bar{e}_{3}\right) ;\left(\bar{e}_{1}, \bar{e}_{2}, \bar{e}_{5}, e_{5}\right), \\
z_{5}^{5} & =\left(e_{1}, e_{2}, e_{3}, \bar{e}_{3}\right) ;\left(\bar{e}_{1}, e_{4}, \bar{e}_{4}, \bar{e}_{2}\right) .
\end{aligned}
$$

Generators for $z_{5}$ are:

$$
\begin{aligned}
& z_{5} e_{1}, z_{5} e_{2}, z_{5} e_{4}, z_{5} e_{5}, \\
& z_{5} e_{1} e_{4}, z_{5} e_{1} e_{5}, z_{5} e_{1} e_{2}, \\
& z_{5} e_{2} e_{1}, z_{5} e_{2} e_{5}, z_{5} e_{2} e_{4}, \\
& z_{5} e_{4} e_{1}, z_{5} e_{4} e_{2}, \\
& z_{5} e_{5} e_{1}, z_{5} e_{5} e_{2} .
\end{aligned}
$$

We see $z_{1}^{3}=g_{5,1}^{1,3} \cdot z_{5}^{1}, z_{1}^{3}=g_{5,1}^{2,3} \cdot z_{5}^{2}$, where

$$
g_{5,1}^{1,3}=\left\{\begin{array}{rl}
e_{2} & \mapsto \bar{e}_{5}, \\
e_{3} & \mapsto \bar{e}_{4}, \\
e_{4} & \mapsto e_{1}, \\
e_{5} & \mapsto e_{2} ;
\end{array} \quad g_{5,1}^{2,3}=\left\{\begin{aligned}
e_{1} & \mapsto e_{1}, \\
e_{3} & \mapsto \bar{e}_{2}, \\
e_{4} & \mapsto e_{5}, \\
e_{5} & \mapsto \bar{e}_{4}
\end{aligned}\right.\right.
$$


Relations for $z_{5}$ are:

$$
\begin{aligned}
& z_{5} e_{1}=1, z_{5} e_{4}=1, z_{5} e_{5}=1, \\
& z_{5} e_{1} e_{4}=z_{1} e_{3} e_{1}, z_{5} e_{1} e_{5}=z_{1} e_{3} e_{2}, z_{5} e_{1} e_{2}=z_{1} e_{2} e_{4}, \\
& z_{5} e_{2} e_{1}=z_{1} e_{3} e_{1}, z_{5} e_{2} e_{5}=z_{1} e_{3} e_{4}, z_{5} e_{2} e_{4}=z_{1} e_{3} e_{5}, \\
& z_{5} e_{1} \cdot z_{5} e_{1} e_{4}=z_{5} e_{4} \cdot z_{5} e_{4} e_{1}, z_{5} e_{1} \cdot z_{5} e_{1} e_{5}=z_{5} e_{5} \cdot z_{5} e_{5} e_{1}, \\
& \quad z_{5} e_{1} \cdot z_{5} e_{1} e_{2}=z_{5} e_{2} \cdot z_{5} e_{3} e_{1}, \\
& z_{5} e_{2} \cdot z_{5} e_{2} e_{5}=z_{5} e_{5} \cdot z_{5} e_{5} e_{2}, z_{5} e_{2} \cdot z_{5} e_{2} e_{4}=z_{5} e_{4} \cdot z_{5} e_{4} e_{2} .
\end{aligned}
$$

An easy simplification shows that neither new generators nor new relations are needed.

From $z_{6}$ we have

$$
\begin{gathered}
z_{6}=\left(e_{1}, e_{2}, e_{3}, \bar{e}_{1}\right) ;\left(\bar{e}_{2}, e_{4}, e_{5}\right),\left(\bar{e}_{3}, \bar{e}_{5}, \bar{e}_{4}\right), \\
z_{6}^{2}=\left(e_{1}, e_{4}, e_{5}, e_{3}, \bar{e}_{1}\right) ;\left(\bar{e}_{3}, \bar{e}_{5}, \bar{e}_{4}\right), \\
z_{6}^{3}=\left(e_{1}, e_{2}, \bar{e}_{5}, \bar{e}_{4}, \bar{e}_{1}\right) ;\left(\bar{e}_{2}, e_{4}, e_{5}\right), \\
z_{6}^{4}=\left(e_{1}, e_{2}, e_{3}, \bar{e}_{1}\right) ;\left(\bar{e}_{2}, \bar{e}_{3}, \bar{e}_{5}, e_{5}\right), \\
z_{6}^{5}=\left(e_{1}, e_{2}, e_{3}, \bar{e}_{1}\right) ;\left(\bar{e}_{2}, e_{4}, \bar{e}_{4}, \bar{e}_{3}\right) .
\end{gathered}
$$

Generators for $z_{6}$ are:

$$
\begin{aligned}
& z_{6} e_{2}, z_{6} e_{3}, z_{6} e_{4}, z_{6} e_{5}, \\
& z_{6} e_{2} e_{4}, z_{6} e_{2} e_{5}, z_{6} e_{2} e_{3}, \\
& z_{6} e_{3} e_{2}, z_{6} e_{3} e_{5}, z_{6} e_{3} e_{4}, \\
& z_{6} e_{4} e_{2}, z_{6} e_{4} e_{3}, \\
& z_{6} e_{5} e_{2}, z_{6} e_{5} e_{3} .
\end{aligned}
$$

We see $z_{2}^{4}=g_{6,2}^{2,4} \cdot z_{6}^{2}, z_{2}^{4}=g_{6,2}^{3,4} \cdot z_{6}^{3}$, where

$$
g_{6,2}^{2,4}=\left\{\begin{array}{rl}
e_{1} & \mapsto e_{1}, \\
e_{3} & \mapsto e_{5}, \\
e_{4} & \mapsto e_{2}, \\
e_{5} & \mapsto e_{3} ;
\end{array} \quad g_{6,2}^{3,4}=\left\{\begin{aligned}
e_{1} & \mapsto e_{1}, \\
e_{2} & \mapsto e_{2}, \\
e_{4} & \mapsto \bar{e}_{5}, \\
e_{5} & \mapsto \bar{e}_{3}
\end{aligned}\right.\right.
$$

Relations for $z_{6}$ are:

$$
\begin{aligned}
& z_{6} e_{2}, z_{6} e_{4}=1, z_{6} e_{5}=1, \\
& z_{6} e_{2} e_{4}=z_{2} e_{4} e_{2}, z_{6} e_{2} e_{5}=z_{2} e_{4} e_{3}, z_{6} e_{2} e_{3}=z_{2} e_{4} e_{5}, \\
& z_{6} e_{3} e_{2}=z_{2} e_{4} e_{2}, z_{6} e_{3} e_{5}=z_{2} e_{4} e_{3}, z_{6} e_{3} e_{4}=z_{2} e_{4} e_{5}, \\
& z_{6} e_{2} \cdot z_{6} e_{2} e_{4}=z_{6} e_{4} \cdot z_{6} e_{4} e_{2}, z_{6} e_{2} \cdot z_{6} e_{2} e_{5}=z_{6} e_{5} \cdot z_{6} e_{5} e_{2}, \\
& \quad z_{6} e_{2} \cdot z_{6} e_{2} e_{3}=z_{6} e_{3} \cdot z_{6} e_{3} e_{2}, \\
& z_{6} e_{3} \cdot z_{6} e_{3} e_{5}=z_{6} e_{5} \cdot z_{6} e_{5} e_{3}, z_{6} e_{3} \cdot z_{6} e_{3} e_{4}=z_{6} e_{4} \cdot z_{6} e_{4} e_{3} .
\end{aligned}
$$


An easy simplification shows that neither new generators nor new relations are needed.

We conclude

$$
\begin{aligned}
\mathcal{A M}_{0,1,3} & =\left\langle z_{1} e_{2} e_{4}, z_{1} e_{3} e_{5}, z_{1} e_{4} e_{5} \mid \begin{array}{l}
z_{1} e_{4} e_{5}=z_{1} e_{2} e_{4} \cdot z_{1} e_{3} e_{5} \cdot z_{1} e_{2} e_{4}, \\
z_{1} e_{4} e_{5} \cdot z_{1} e_{3} e_{5}=z_{1} e_{2} e_{4} \cdot z_{1} e_{4} e_{5}
\end{array}\right\rangle \\
& =\left\langle z_{1} e_{2} e_{4}, z_{1} e_{3} e_{5} \mid z_{1} e_{3} e_{5} \cdot z_{1} e_{2} e_{4} \cdot z_{1} e_{3} e_{5}=z_{1} e_{2} e_{4} \cdot z_{1} e_{3} e_{5} \cdot z_{1} e_{2} e_{4}\right\rangle .
\end{aligned}
$$

\section{Acknowledgments}

I am grateful to Warren Dicks for introducing me to algebraic mapping-class groups and to Luis Paris for his advices during a post-doc in Dijon.

\section{References}

[1] Heather Armstrong, Bradley Forrest, and Karen Vogtmann. A presentation for $\operatorname{Aut}\left(F_{n}\right)$. J. Group Theory, 11(2):267-276, 2008.

[2] Heather Armstrong, Bradley Forrest, and Karen Vogtmann. A presentation for $\operatorname{Aut}\left(F_{n}\right)$. J. Group Theory, 11(2):267-276, 2008.

[3] Silvia Benvenuti. Finite presentations for the mapping class group via the ordered complex of curves. Adv. Geom., 1(3):291-321, 2001.

[4] Joan S. Birman and Hugh M. Hilden. On the mapping class groups of closed surfaces as covering spaces. In Advances in the theory of Riemann surfaces (Proc. Conf., Stony Brook, N.Y., 1969), pages 81-115. Ann. of Math. Studies, No. 66. Princeton Univ. Press, Princeton, N.J., 1971.

[5] Kenneth S. Brown. Presentations for groups acting on simply-connected complexes. $J$. Pure Appl. Algebra, 32(1):1-10, 1984.

[6] Marc Culler and Karen Vogtmann. Moduli of graphs and automorphisms of free groups. Invent. Math., 84(1):91-119, 1986.

[7] Warren Dicks and Edward Formanek. Automorphism subgroups of finite index in algebraic mapping class groups. J. Algebra, 189(1):58-89, 1997.

[8] Warren Dicks and Edward Formanek. Algebraic mapping-class groups of orientable surfaces with boundaries. In Infinite groups: geometric, combinatorial and dynamical aspects, volume 248 of Progr. Math., pages 57-116. Birkhäuser, Basel, 2005.

[9] Sylvain Gervais. A finite presentation of the mapping class group of a punctured surface. Topology, 40(4):703-725, 2001.

[10] John Harer. The second homology group of the mapping class group of an orientable surface. Invent. Math., 72(2):221-239, 1983.

[11] A. Hatcher and W. Thurston. A presentation for the mapping class group of a closed orientable surface. Topology, 19(3):221-237, 1980.

[12] Susumu Hirose. A complex of curves and a presentation for the mapping class group of a surface. Osaka J. Math., 39(4):795-820, 2002. 
[13] Catherine Labruère and Luis Paris. Presentations for the punctured mapping class groups in terms of Artin groups. Algebr. Geom. Topol., 1:73-114 (electronic), 2001.

[14] Makoto Matsumoto. A presentation of mapping class groups in terms of Artin groups and geometric monodromy of singularities. Math. Ann., 316(3):401-418, 2000.

[15] James McCool. Some finitely presented subgroups of the automorphism group of a free group. J. Algebra, 35:205-213, 1975.

[16] James McCool. Generating the mapping class group (an algebraic approach). Publ. Mat., 40(2):457-468, 1996.

[17] Bronislaw Wajnryb. A simple presentation for the mapping class group of an orientable surface. Israel J. Math., 45(2-3):157-174, 1983.

[18] Bronislaw Wajnryb. Artin groups and geometric monodromy. Invent. Math., 138(3):563-571, 1999. 